\title{
Anti-inflammatory mechanisms of suppressors of cytokine signaling target ROS via NRF-2/thioredoxin induction and inflammasome activation in macrophages
}

\author{
Ga-Young Kim", Hana Jeong ${ }^{\#}$, Hye-Young Yoon, Hye-Min Yoo, Jae Young Lee, Seok Hee Park E Choong-Eun Lee \\ Department of Biological Science, College of Science, Sungkyunkwan University, Suwon 16419, Korea
}

\begin{abstract}
Suppressors of cytokine signaling (SOCS) exhibit diverse antiinflammatory effects. Since ROS acts as a critical mediator of inflammation, we have investigated the anti-inflammatory mechanisms of SOCS via ROS regulation in monocytic/macrophagic cells. Using PMA-differentiated monocytic cell lines and primary BMDMs transduced with SOCS1 or shSOCS1, the LPS/TLR4induced inflammatory signaling was investigated by analyzing the levels of intracellular ROS, antioxidant factors, inflammasome activation, and pro-inflammatory cytokines. The levels of LPS-induced ROS and the production of pro-inflammatory cytokines were notably down-regulated by SOCS1 and up-regulated by shSOCS1 in an NAC-sensitive manner. SOCS1 up-regulated an ROS-scavenging protein, thioredoxin, via enhanced expression and binding of NRF-2 to the thioredoxin promoter. SOCS3 exhibited similar effects on NRF-2/thioredoxin induction, and ROS downregulation, resulting in the suppression of inflammatory cytokines. Notably thioredoxin ablation promoted NLRP3 inflammasome activation and restored the SOCS1-mediated inhibition of ROS and cytokine synthesis induced by LPS. The results demonstrate that the anti-inflammatory mechanisms of SOCS1 and SOCS3 in macrophages are mediated via NRF-2-mediated thioredoxin upregulation resulting in the downregulation of ROS signal. Thus, our study supports the anti-oxidant role of SOCS1 and SOCS3 in the exquisite regulation of macrophage activation under oxidative stress. [BMB Reports 2020; 53(12): 640-645]
\end{abstract}

\section{INTRODUCTION}

Inflammation is a front-line defense mechanism triggered by expo-

*Corresponding author. Tel: +82-31-290-7006; Fax: +82-31-290-7015; E-mail: celee@skku.edu

${ }^{\#}$ These authors contributed equally to this work.

https://doi.org/10.5483/BMBRep.2020.53.12.161

Received 31 July 2020, Revised 6 September 2020, Accepted 22 October 2020

Keywords: Inflammasome, Reactive oxygen species (ROS), Suppressors of cytokine signaling (SOCS), Thioredoxin, TLR4 signal sure to various antigens and involves primarily macrophages, neutrophils and dendritic cells. These cells recognize pathogens with pattern recognition receptors such as TLRs, perform phagocytosis, and regulate the activation of other cells by producing inflammatory cytokines (1).

The generation of reactive oxygen species (ROS) is a key feature of phagocytic cells via oxidative burst, which mediates many aspects of inflammatory reactions (2). While ROS are essential for anti-microbial defense via production of pro-inflammatory cytokines, they have been implicated in diverse inflammatory and autoimmune diseases $(3,4)$. ROS regulation is thus considered important in immune homeostasis. Indeed, ROS broadly participate in the modulation of immune responses via activation of cellular signaling pathways involving tyrosine kinases, tyrosine phosphatases, mitogen-activated protein kinases (MAPK), and NF-KB $(5,6)$. However, the biological mechanisms underlying these processes are not always clear (7).

Lipopolysacharride (LPS) is a principal active agent, which induces a strong inflammatory response by stimulating monocytes and macrophages. LPS is recognized by TLR4, which then triggers MyD88- and TRIF-dependent pathways, leading to the activation of transcription factors $\mathrm{NF}-\mathrm{KB}$ and IFN regulatory factor 3 (IRF3), respectively, resulting in the production of inflammatory cytokines including TNF- $\alpha$, IL-1, IL-6 and IFNs $(8,9)$.

Suppressor of cytokine signaling 1 (SOCS1) was first characterized as a regulator of IFN- $\gamma$-induced inflammatory responses, via inhibition of Jak/STAT pathways (10). SOCS1 expression is increased in macrophages following LPS exposure, and represents a negative feed-back mechanism of LPS signaling (11, 12). The inhibition of NF- $\kappa B$, as well as the downregulation of MyD88-associated adaptor (MAL) and TRAF6 by SOCS1 via SOCS box-mediated protein degradation have been suggested as possible modes of SOCS action regulating the LPS response (13). However, the molecular mechanisms underlying SOCS1 regulation of pro-inflammatory cytokine production appear rather complex involving both direct and indirect mechanisms (14).

We have previously reported the inhibitory action of SOCS1 on ROS-mediated signaling pathways during T cell apoptosis in response to TNF- $\alpha$ and oxidative stress (15). The anti-apoptotic activity of SOCS1 was demonstrated using both over-expression and knock-down systems, via protection of protein tyrosine 
phosphatases as well as direct interaction with Jaks required for the apoptosis signaling. SOCS1 was induced as a protective response to ROS-generating apoptotic stimuli, which represented an intracellular defense mechanism against oxidative stress (15).

Since ROS also acts as a critical mediator of inflammatory signaling, we have investigated the anti-inflammatory mechanism of SOCS action in macrophages during LPS/TLR4 signal via ROS regulation. Our data indicate that SOCS1 and SOCS3 attenuate LPS/TLR4 signals leading to the induction of pro-inflammatory cytokines via ROS downregulation. Specifically, the inhibition of inflammasome activation and induction of thioredoxin were suggested as mechanisms of SOCS action involving ROS suppression to inhibit IL-1 $\beta$ and IL-6, respectively. We further demonstrate that the SOCS1-mediated upregulation of thioredoxin expression involves NRF-2 transcription factor and its binding to the thioredoxin promoter. Taken together, the results of the present study strongly suggest ROS downregulation as a mechanism of anti-inflammatory action of SOCS during macrophage activation.

\section{RESULTS}

SOCS1 down-regulates intracellular ROS to suppress LPS/TLR4 signaling for inflammatory cytokine production In order to investigate the regulation mechanism of SOCS action in inflammatory signaling, human monocytic cell lines (THP1) expressing inflammatory receptors (e.g., TLRs) were used to establish SOCS1 over-expression or knock-down systems. Initially THP1 cells were subjected to viral transduction of Flag (mock) or FlagSOCS1 construct using retroviral vectors. Cells were then treated with PMA to induce differentiation prior to stimulation with LPS, a TLR4 agonist. While both mock and SOCS1-transduced cells responded to PMA by up-regulating TLR4 expression, they exhibited similar levels of surface TLR4 regardless of SOCS1 transduction (Supplementary Fig. 1). As LPS-induced ROS generation is thought to play a key role in the initiation of inflammatory signaling, we have determined the intracellular ROS levels upon LPS stimulation. The kinetics revealed that ROS levels induced by LPS peaked in 15 to $30 \mathrm{~min}$, followed by eventual decline in 60 min. Notably, SOCS1-transduced cells showed a substantial decrease in ROS levels when compared with mock cells (Fig. 1A). LPS then induced production of inflammatory cytokines such as TNF- $\alpha$, IL-1 $\beta$ and IL- 6 by $24 \mathrm{~h}$. Upon SOCS1 over-expression, the production of these cytokines was prominently reduced by $40 \%$ to $70 \%$ (Fig. $1 \mathrm{~B}$ ).

In contrast to cells over-expressing SOCS1, the SOCS1-ablated cells exhibited significantly elevated ROS levels. Both the basal and LPS-induced ROS levels were upregulated by shSOCS1 transduction, which was abrogated by treatment with an anti-oxidant NAC (Fig. 1C), indicating that SOCS1 negatively regulates cellular ROS levels. The shSOCS1 cells induced a 2- to 3-fold increase in TNF- $\alpha$, IL-1 $\beta$ and IL- 6 levels compared with mock (sh) cells. NAC not only impaired LPS-induced pro-inflammatory cytokine production in mock cells, but also almost com-

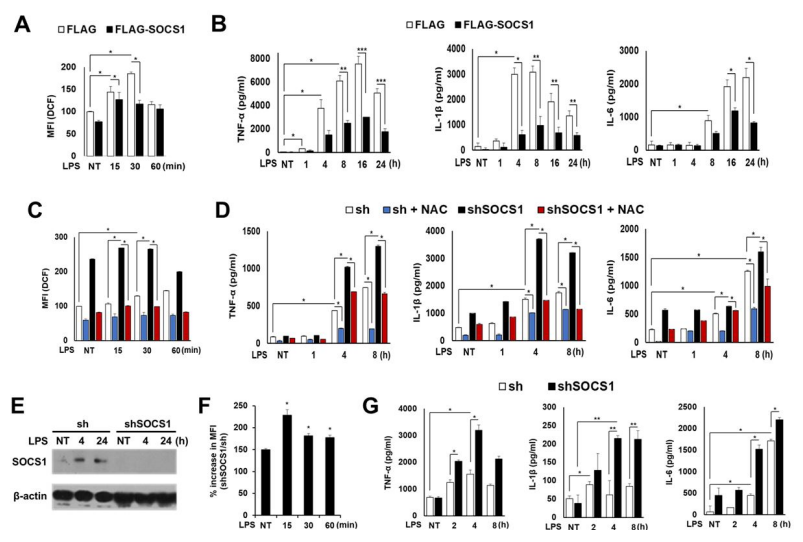

Fig. 1. Inhibitory effects of SOCS1 on pro-inflammatory cytokine production and ROS generation induced by LPS in THP1 human monocytic cells and mouse BMDMs. Flag or Flag-SOCS1 THP1 cells were differentiated by PMA. Cells were then either not treated (NT) or treated with LPS $(1 \mu \mathrm{g} / \mathrm{ml})$ for the indicated duration. ROS levels were determined (A). The culture supernatants were analyzed to measure inflammatory cytokines by ELISA (B). The sh control and shSOCS1-transduced THP1 cells were stimulated with LPS with or without NAC pretreatment, after which ROS $(\mathrm{C})$ and cytokine levels (D) were determined. BMDMs from C57BL/6 mice were transduced with mouse shSOCS1. The SOCS1 levels were analyzed by western blot (E). ROS levels in shSOCS1 cultures were analyzed as \% increase over mock (sh) cultures (F). LPSinduced cytokine production was determined by ELISA (G). Results represent means + SD obtained from three independent experiments performed in triplicate $\left({ }^{*} P<0.05 ;{ }^{+}\right.$n.s. $)$.

pletely blocked the excessive cytokine production in shSOCS1 cells by 4 to $8 \mathrm{~h}$ (Fig. 1D). The data indicate that enhanced ROS levels observed in SOCS1-ablated cells may be responsible for the increased production of inflammatory cytokines. The inhibitory effect of SOCS1 on LPS-induced ROS generation and cytokine production in primary cells was also analyzed in bone marrow-derived macrophages (BMDMs) isolated from C57BL/6 mice. The shSOCS1-transduced BMDM cultures exhibited increased ROS levels compared with mock cultures. As seen in THP1 cells, the LPS-induced inflammatory cytokine levels were generally enhanced in SOCS1-ablated BMDMs (Fig. $1 \mathrm{E}-\mathrm{G})$.

The role of MAPKs and NF- $\kappa B$ has been widely suggested in the principal signaling pathways evoked by LPS/TLR4 for proinflammatory cytokine production $(16,17)$. We found that the early induction of $p$-Erk, p-Jnk, and p-p38 by LPS in THP1 cells was reduced in SOCS1-expressing cells (Supplementary Fig. $2 \mathrm{~A})$. The NF-kB activation, indicated by the peak nuclear p65 levels after $30 \mathrm{~min}$, was also significantly down-regulated by SOCS1 transduction. In addition, phosphoserine (pS)-STAT1 and phosphotyrosine (pY)-STATs 1,3 and 5 induced by LPS from 30 to $240 \mathrm{~min}$, were significantly suppressed in SOCS1-overexpressing cells (Supplementary Fig. 2A). In contrast, the LPSinduced activation of MAPKs, NF- $\mathrm{KB}$, and STATs was promoted by SOCS1 ablation and completely inhibited by NAC, which 
again suggests ROS as a SOCS1 target during LPS-induced inflammation signaling (Supplementary Fig. 2B).

\section{The anti-oxidant NRF-2 and thioredoxin are selectively upregulated by SOCS1}

The above data indicate that the downregulation of intracellular ROS by SOCS1 contributes to the anti-inflammatory effects of SOCS1 in both monocytic/macrophagic cell lines and primary macrophages, suggesting that ROS-scavenging proteins mediate the action of SOCS1. Analysis of various anti-oxidant enzymes revealed that thioredoxin is the primary target regulated by SOCS1 and shSOCS1 (Fig. 2A and 2B). While LPS stimulation of mock cells induced a gradual increment in thioredoxin mRNA and protein levels, the basal levels of thioredoxin in SOCS1transdued cells were up-regulated and maintained at increased levels upon LPS stimulation (Fig. 2A and 2C). In contrast, the levels of other anti-oxidant enzymes such as catalase, SOD1, and peroxidase were not significantly affected by SOCS1 or shSOCS1.

Next, to investigate the mechanism of thioredoxin upregulation by SOCS1, the activation of NRF-2 transcription factor implicated in the induction of anti-oxidant response genes was analyzed (18). We have noted that NRF-2 levels were elevated by SOCS1 over-expression, similar to increased thioredoxin levels (Fig. 2D). Furthermore, the CHIP assay revealed that while NRF-2 binding to the anti-oxidant response element (ARE) of thioredoxin promoter was gradually increased by $60 \mathrm{~min}$ of LPS treatment in mock cells, its binding was notably enhanced in Flag-SOCS1 cells and maintained at high levels (Fig. 2E). The results suggest that SOCS1 exhibits ROS-scavenging effects by inducing thioredoxin mediated by the action of NRF-2 antioxidant transcription factor.

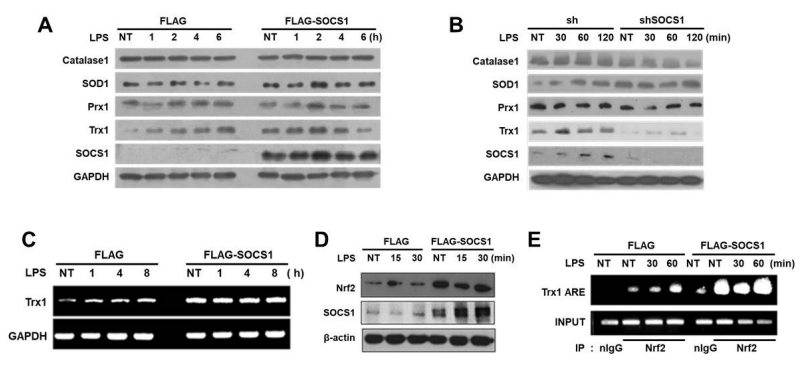

Fig. 2. Thioredoxin expression is upregulated by SOCS1 via increased NRF-2 binding to the ARE of the thioredoxin promoter. THP1 cells transduced with SOCS1 or shSOCS1 were stimulated with LPS and the expression levels of antioxidant proteins were analyzed by immunoblotting (A, B). Thioredoxin levels were confirmed in SOCS1-transduced cells by RT-PCR (C). NRF-2 levels and NRF-2 binding to the ARE of the thioredoxin promoter were assessed by immunoblotting and CHIP assays as described in Supplementary Materials (D, E).
SOCS3 exhibits similar inhibitory effects on LPS-induced ROS and inflammatory cytokine production

We have also analyzed the effect of SOCS3 on LPS-induced ROS signaling leading to inflammation response. Although SOCS1 and SOCS3 share common structural features, they exhibit cell type-dependent expression patterns with distinct roles in inflammation (19).

The expression level of SOCS3 is found low in monocytes and macrophages both in vitro and in vivo, unless exposed to inflammatory stimuli $(20,21)$. While SOCS3 is induced by LPS stimulation within $1 \mathrm{~h}$ in mock THP1 cells, the SOCS3-tranduced cells show increased basal SOCS3 levels, which were maintained during LPS treatment. In these cells, a significant downregulation of LPS-induced ROS levels was correlated with increased expression of NRF2 and TRX1 within $1 \mathrm{~h}$ (Fig. 3A and $3 \mathrm{~B})$. As in the case for SOCS1, the production of inflammatory cytokines such as TNF- $\alpha$, IL-1 $\beta$ and IL- 6 was strongly suppressed in SOCS3-over-expressing cells (Fig. 3C). Therefore, similar to SOCS1, SOCS3 exhibits prominent inhibitory effects on inflammatory signaling during ROS regulation in response to LPS.

\section{Role of thioredoxin in SOCS1-mediated ROS regulation and the suppression of LPS/ROS-induced inflammasome activation by SOCS1}

To evaluate whether the increase in thioredoxin levels is responsible for ROS reduction in SOCS over-expressing cells, we investigated the effect of thioredoxin transduction. First, it was observed that thioredoxin gene transduction per se resulted in the suppression of LPS-induced early ROS generation (Supplementary
A

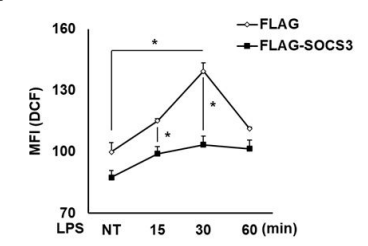

B

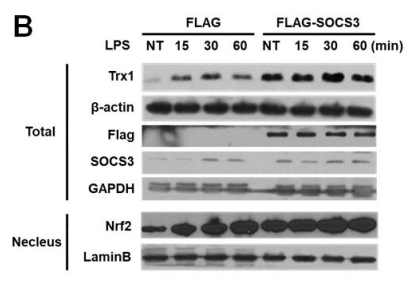

C
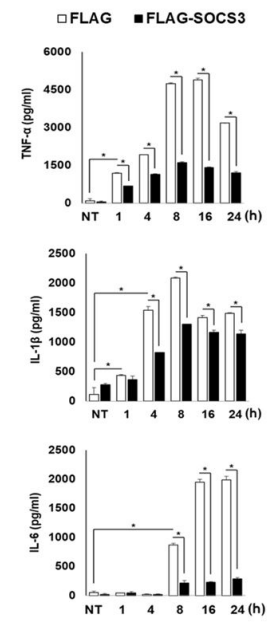

Fig. 3. Suppressive effects of SOCS3 on LPS-induced ROS and inflammatory cytokine production accompanied by increased NRF-2 and thioredoxin expression. THP1 cells transduced with Flag or Flag-SOCS3 were analyzed for LPS-induced intracellular ROS (A), NRF-2, thioredoxin and SOCS3 levels by Western blot (B), and inflammatory cytokine production by ELISA (C). 
Fig. 3A). Next, to determine the role of thioredoxin in mediating SOCS1 action, the effect of thioredoxin knock-down on LPS-induced ROS generation and cytokine production was analyzed. While Flag-SOCS1 cells exhibited increased thioredoxin and reduced ROS levels, thioredoxin ablation with shTrx introduction into these cells triggered a robust increase in ROS generation by LPS at 30 min (Fig. 4A). The synthesis of inflammatory cytokine IL-6 in both Flag and Flag-SOCS1 cells was upregulated by thioredoxin depletion. Notably, the IL-6 level was restored completely in Flag-SOCS1 cells upon thioredoxin ablation both at 4 and $8 \mathrm{~h}$ after LPS stimulation (Supplementary Fig. 3B). The result suggests a critical role of thioredoxin in SOCS1mediated suppression of LPS-induced ROS and cytokine production.

The production of inflammatory cytokines in activated macrophages by pathogen sensing occurs via inflammasome assembly, a multi-molecular complex required for caspase 1 activation (22). As inflammasome activation is triggered by ROS (23), we examined the effect of thioredoxin on LPS-induced inflammasome activation leading to caspase 1 cleavage for IL-1 $\beta$ maturation. NLRP3 inflammasome was first analyzed in LPS-primed cells by ROS induction with nigericin. The shTrx-transduced cells exhibited a substantial increase in LPS-induced NLRP3 levels, which were further promoted by nigericin. The ASC oligomer formation was induced by LPS and enhanced by nigericin treatment. Subsequently, the shTRX1 transduction promoted the inflammasome assembly leading to caspase- 1 cleavage and IL-1 $\beta$ production, indicating that thioredoxin controls LPS/ROS-induced NLRP3 inflammasome activation (Fig. 4B and 4C).
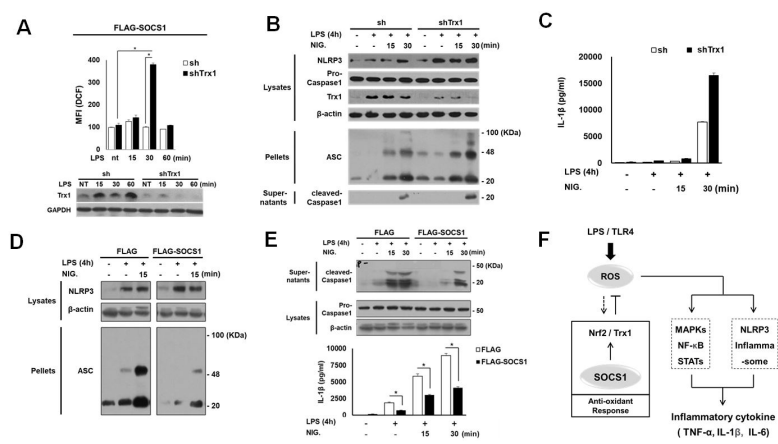

Fig. 4. Role of thioredoxin in SOCS1-induced suppression of LPS response for ROS generation and inflammasome activation associated with IL-1 $\beta$ production. Flag-SOCS1 THP1 cells were further transfected with sh/shTrx1 and the intracellular ROS levels were analyzed following LPS stimulation (A). The role of Trx1 and SOCS1 in LPS-induced, ROS-promoted inflammasome activation was analyzed. Cells were stimulated with LPS for $4 \mathrm{~h}$ and treated with nigericin (NIG) as indicated. Activation of ASC, NLRP3 and caspase-1 was then analyzed by immunoblotting and the secreted IL-1 $\beta$ protein was measured by ELISA (B-E). The proposed model represents the inhibitory mechanism of SOCS1 in TLR4 signaling leading to inflammatory cytokine production via downregulation of ROS (F). The details are described in Supplementary Materials.
Next, we investigated the possibility that inflammasome activation downstream of TLR4 signaling is regulated by SOCS1. Flag-SOCS1 cells exhibited a significant attenuation of ASC protein synthesis and the ASC oligomer formation induced by the NLRP3 stimulant nigericin (Fig. 4D). This inhibition of assembly correlated strongly with a lack of caspase- 1 maturation into its processed 20-kDa form and suppression of IL-1 $\beta$ production (Fig. 4E). The data indicate that both SOCS1 and thioredoxin counteract the LPS-induced inflammasome activation likely via ROS regulation.

\section{DISCUSSION}

The present work demonstrates the anti-oxidant function of SOCS in the regulation of inflammatory signaling. Both SOCS1 and SOCS3 increased thioredoxin expression and down-regulated the basal and LPS-induced early ROS generation to attenuate the expression of pro-inflammatory cytokines. The results are consistent with our earlier findings of thioredoxin-promoting effect of SOCS1 resulting in the suppression of the ROS signalinduced apoptosis of Jurkat T cells (15). In the present study, we have further investigated the molecular mechanism of thioredoxin upregulation by SOCS1. As an anti-oxidant defense gene, thioredoxin is considered a target of anti-oxidant transcription factor NRF-2 which binds to the ARE sequence of diverse genes induced under oxidative stress $(18,24)$. We have in fact identified an ARE-related sequence in the thioredoxin promoter and performed CHIP assays using primers designed to dectect NRF-2 binding. The increases in the basal NRF-2 level and its binding to the thioredoxin promoter correlate with the upregulation of the basal thioredoxin expression in Flag-SOCS1 cells (Fig. 2A and 2C). Currently, the mechanism of NRF-2 upregulation by SOCS1 and SOCS3 is not clear. As NRF-2 level is subject to downregulation by iNRF-2 (Keap1)-mediated degradation, SOCS1 and SOCS3 may competitively inhibit Keap1 action via SOCS-box to bind with Culin/Rbx/E2 ligase required for ubiqutination of NRF-2 (25). The increased basal thioredoxin via NRF-2 upregulation in SOCS1- or SOCS3-transduced cells likey sustains the ROS downregulation in the initial phase of LPS signaling. Such ROS regulation represents an upstream signaling event, which is critical for the downstream response including the production of inflammatory cytokines.

SOCS3 shares common properties with SOCS1 including the structural domains such as KIR, SH2 and SOCS boxes, although their reported functions are often distinct. For example, in contrast to SOCS1, which inhibits the T cell apoptosis induced by hydrogen peroxide and TNF- $\alpha$ via ROS downregulation, SOCS3 promoted the oxidant-induced T cell apoptosis (15). However, in this work, both SOCS1 and SOCS3 strongly inhibited the inflammatory cytokine production involving ROS suppression within 30 min of LPS stimulation. Compared with cells transduced with either SOCS1 or SOCS3, cells transduced with both SOCS1 and SOCS3 exhibited a further inhibition of IL-1 and IL-6 production (Supplementary Fig. 4A), suggesting that SOCS1 
and SOCS3 co-operate to down-regulate LPS-induced ROS signaling in inflammatory response. It may also indicate that the two SOCS isoforms utilize distinct additional pathways to synergistically suppress inflammatory signaling. Although both SOCS1 and SOCS3 have been reported to modulate apoptosis induced by ROS and Fas ligation in Jurkat T cells $(15,26)$, cell death was not affected by SOCS1 or SOCS3 in THP1 cells during LPS treatment (Supplementary Fig. 4B). This finding excludes the possibility that SOCS-mediated suppression of cytokine production occurs due to increased cell death.

The current study also revealed that SOCS1 acts as a potent inhibitor of ROS-mediated NLRP3 inflammasome activation required for caspase 1 activation (Fig. 4). Thus, the anti-inflammatory action of SOCS1 encompasses ROS signal-dependent cytokine induction and processing, which leads to the regulated production of cytokine cascade. The suppressive effect of SOCS1 on inflammasome activation contrasts with the effect of thioredoxin ablation, which promoted the LPS-induced ROS-mediated NLRP3 inflammasome. The data again support the anti-inflammatory mechanism of SOCS1 via thioredoxin induction.

In summary, SOCS1 effectively suppresses LPS-induced signaling and NLRP3 inflammasome by down-regulating intracellular ROS levels via NRF-2/thioredovxin induction (Fig. 4F). During inflammation, SOCS1 is directly induced by ROS upon LPS stimulation $(15,27)$. SOCS may also be induced by the cytokines produced downstream of LPS signaling as these cells express receptors for inflammatory cytokines (Supplementary Fig. 5). However, the kinetics of ROS-dependent STAT activation observed upstream of cytokine production (Supplementary Fig. 2B) suggest the induction of SOCS in a proximal negative feedback of LPS signaling. The SOCS then likely induces anti-oxidant response for removal of excess ROS triggered by the inflammation. Upregulation of thioredoxin reduces ROS levels (24) and apparently contributes to the anti-inflammatory action of SOCS in macrophages. Since elevated thioredoxin expression is correlated with increased NRF-2 levels in SOCS-transduced cells, the molecular mechanism by which SOCS upregulates anti-oxidant transcription factor NRF-2 in inflammatory cells warrants further investigation. Such studies are needed to establish the novel function of SOCS as a member of anti-oxidant defense system and its potential therapeutic value in inflammatory diseases caused by ROS deregulation.

\section{MATERIALS AND METHODS}

\section{Cell culture and generation of SOCS over-expressing or knock-down cell systems}

Human acute monocytic leukemia cell lines (THP1) were maintained in RPMI media containing 10\% FBS (Invitrogen, Carsbed) and cultured in a humidified $5 \% \mathrm{CO}_{2}$ incubator. The preparation of bone marrow-derived macrophages (BMDMs) from C57BL/6 mice (28) and the viral transduction of SOCS1, SOCS3 and $\operatorname{Tr} 1$ genes or sh constructs were performed as detailed in the Supplementary Materials. PMA (100 ng/ml, 16 h)-treated monocytic cells and BMDMs were stimulated by $1 \mu \mathrm{g} / \mathrm{ml}$ LPS (E.Coli 0111:B4, Sigma-Aldrich). Treatment with anti-oxidant NAC ( $1 \mathrm{mM})$ was carried out $1 \mathrm{~h}$ prior to LPS stimulation.

\section{Determination of intracellular ROS by FACS}

Cells were stimulated with LPS for indicated durations and a redox-sensitive cell permeable dye $\mathrm{H}_{2}$ DCF-DA $(1 \mu \mathrm{M})$ was added to cultures $20 \mathrm{~min}$ before the end of the incubation. Cells were simultaneously harvested and DCF fluorescence was measured at $530 \mathrm{~nm}$ to assess intracellular ROS levels, using FACS Caliber (BD Bioscience, Mountainview, CA).

\section{Cell fractionation and westem blot analysis}

LPS-primed cells were lysed to prepare total, cytosolic and nuclear extracts (26). The proteins were resolved by SDS-PAGE and transferred to PVDF membranes for immunoblotting using the antibodies described in Supplementary Materials.

\section{Measurement of cytokines by ELISA}

TNF- $\alpha$, IL- $1 \beta$ and IL- 6 levels were assessed using human and mouse cytokine ELISA kits (e-Bioscience and R\&D Systems).

\section{RT-PCR and qRT-PCR}

Cells were harvested for total RNA extraction using TRIzol. RT-PCR of thioredoxin was performed as described (15). The qRT-PCR amplification with POWER SYBR ${ }^{\circledR}$ Green (Applied Biosystems, Warrington, UK) was performed using a Mastercycler realplex thermalcylcer (Eppendorf AG, Hamburg, Germany).

\section{Determination of inflammasome activity}

Inflammasome assays were performed in two stages: LPS priming for $4 \mathrm{~h}$ and inflammasome activation within $1 \mathrm{~h}$ to avoid any chemical induction at the LPS priming stage as described in the Supplementary Materials.

\section{Statistical analysis}

Experiments were conducted using at least 3 independent sets in duplicate or triplicate cultures. The values are represented as means \pm SD. Staistical significance was determined via Student's $t$ test. A value of $\mathrm{P}<0.05$ was considered statistically significant.

\section{ACKNOWLEDGEMENTS}

This study is supported by National Research Foundation of Korea (NRF) Grants \#2012R1A2A2A01015258, \#2015M2B2A9029226, and \#2018R1A2B6002201. Hana Jeong is supported in part by NRF-2017-Global Ph.D. Fellowship Program.

\section{CONFLICTS OF INTEREST}

The authors have no conflicting interests. 


\section{REFERENCES}

1. Takeda K and Akira S (2005) Toll-like receptors in innate immunity. Int Immunol 17, 1-14

2. Kohchi Cl, Inagawa H, Nishizawa T and Soma G (2009) ROS and innate immunity. Anticancer Res 29, 817-821

3. Filippin LI, Vercelino R, Marroni NP and Xavier RM (2008) Redoxsignalling and the inflammatory response in rheumatoid arthritis. Clin Exp Immunol 152, 415-422

4. Gilgun-Sherki Y, Melamed E and Offen D (2004) The role of oxidativestress in the pathogenesis of multiple sclerosis: the need for effective antioxidanttherapy. J Neurol 251, 261268

5. Nathan C (2003) Specificity of a third kind: reactive oxygen and nitrogen intermediates in cell signaling. J Clin Invest $111,769-778$

6. Kamata H, Honda S, Maeda S, Chang L, Hirata H and Karin, M (2005) Reactive oxygen species promote TNF alpha-induced death and sustained JNK activation by inhibiting MAP kinase phosphatases. Cell 120, 649-661

7. Ray PD, Huang BW and Tsuji Y (2012) Reactive oxygen species (ROS) homeostasis and redox regulation in cellular signaling. Cell Signal 24, 981-990

8. Medzhitov R, Preston-Hurlbrt P, Kopp E et al (1998) MyD88 is an adaptor protein in the hToll/IL-1 receptor family signaling pathways. Mol Cell 2, 253-258

9. Muzio M, Natoli G, Saccani S, Levrero M and Mantovani A (1998) The human toll signaling pathway: divergence of nuclear factor kappaB and JNK/SAPK activation upstream of tumor necrosis factor receptor-associated factor 6 (TRAF6). J Exp Med 187, 2097-2101

10. Alexander WS, Starr R, Fenner JE et al (1999) SOCS1 is a critical inhibitor of interferon gamma signaling and prevents the potentially fatal neonatal actions of this cytokine. Cell 98, 597-608

11. Nakagawa R, Naka T, Tsutsui $\mathrm{H}$ et al (2002) SOCS-1 participates in negative regulation of LPS responses. Immunity 17 , 677-687

12. Kimura A, Naka T, Muta T et al (2005) Suppressor of cytokine signaling-1 selectively inhibits LPS-induced IL-6 production by regulating JAK-STAT. Proc Natl Acad Sci U S A 102, 17089-17094

13. Mansell A, Smith R, Doyle SL et al (2006) Suppressor of cytokine signaling 1 negatively regulates Toll-like receptor signaling by mediating Mal degradation. Nat Immunol 7, 148-155

14. Baetz A, Frey M, Heeg K and Dalpke AH (2004) Suppressor of cytokine signaling (SOCS) proteins indirectly regulate toll-like receptor signaling in innate immune cells. J Biol
Chem 279, 54708-54715

15. Oh J, Hur MW and Lee CE (2009) SOCS1 protects protein tyrosine phosphatases by thioredoxinupregulation and attenuates Jaks to suppress ROS-mediated apoptosis. Oncogene 28, 3145-3156

16. Yamamoto M, Yamazaki S, Uematsu S et al (2004) Regulation of Toll/IL-1-receptor-mediated gene expression by the inducible nuclear protein IkappaBzeta. Nature 430, 218-222

17. Ryan KA, Smith MF Jr, Sanders MK and Emst PB (2004) Reactive oxygen and nitrogen species differntially regulate Toll-like receptor 4-mediated activation of NF-kappa B and interleukin-8 expression. Infect Immun 72, 2123-2130

18. Anuranjani and Bala M (2014) Concerted action of Nrf2-ARE pathway, MRN complex, HMGB1 and inflammatory cytokines: Implication in modification of radiation damage. Redox Biol 2, 832-846

19. Duncan SA, Baganizi DR, Sahu R, Singh SR and Dennis VA (2017) SOCS proteins as regulators of inflammatory responses induced by bacterial infections: A review. Front Microbiol 8, 2431

20. Lui Y, Stewart KN, Bishop E et al (2008) Unique expression of suppressor of cytokine signaling 3 is essential for classical macrophage activation in rodents in vitro and in vivo. J Immunol 180, 6270-6278

21. Qin H, Holdbrooks T, Li Y et al (2012) SOCS3 Deficiency promotes M1 macrophage polarization and inflammation. J Immunol 189, 3439-3448

22. Schroder K and Tschopp J (2010) Theinflammasomes. Cell $140,821-832$

23. Yang $Y$, Wang $H$, Kouadir $M$, Song $H$ and Shi $F$ (2019) Recent advances in the mechanisms of NLRP3 inflammasome activation and its inhibitors. Cell Death Dis 10, 128

24. Lu J and Holmgren A (2014) The thioredoxin antioxidant system. Free Radical Biol Med 66, 75-87

25. Kobayashi A, Kang MI, Okawa $\mathrm{H}$ et al (2004) Oxidative stress sensor Keap1 functions as an adaptor for Cul3-based E3 ligase to regulate proteasomal degradation of $\mathrm{Nrf}$. Mol Cell Biol 24, 7130-7139

26. Oh J, Kim SH, Ahn S and Lee CE (2012) Suppressors of cytokine signaling promote Fas-induced apoptosis by downregulation of $\mathrm{NF}-\mathrm{\kappa B}$ and mitochondrial Bfl-1 in leukemic $\mathrm{T}$ cells. J Immunol 189, 5561-5571

27. Kim SY, Jeong J-M, Kim SJ et al (2017) Pro-inflammatory hepatic macrophages generate ROS through NADPH oxidase 2 via endocytosis of monomeric TLR4-MD2 complex. Nat Commun 8, 2247

28. Zhang X, Goncalves R and Mosser DM (2008) The isolation and characterization of murine macrophages. Curr Proc Immunol 83, 14.1.1-14.1.14 